\title{
Avaliação de parâmetros operacionais em um ga- seificador de biomassa de fluxo concorrente (downdraft ventilado)
}

Loureiro, E. C. M.

Escola Politécnica de Pernambuco Universidade de Pernambuco 50.720-001 - Recife, Brasil Eduloureiro@uol.com

\author{
Garrett, I. A. 2 \\ Escola Politécnica de Pernambuco \\ Universidade de Pernambuco \\ 50.720-001 - Recife, Brasil \\ isabellagarrett@hotmail.com
}

\begin{abstract}
Resumo O projeto "Avaliações de parâmetros operacionais em um gaseificador de biomassa de fluxo concorrente", que teve início na primeira semana de outubro de 2011, visa avaliar a influência dos diversos tipos de biomassa e dos parâmetros operacionais de um gaseificador do tipo fluxo concorrente (downdraft). Assim como, realizar coletas e análise quantitativa e qualitativa das amostras com a finalidade de promover melhorias na pesquisa e com isso aprimora essa tecnologia. Bem como, estudar a aplicação do gás produzido em motores de combustão interna e a sua utilização no setor elétrico. Essa pesquisa proporciona um maior entendimento sobre a gaseificação de biomassa que não é um processo recente, porém está em contínuo crescimento. Esta tecnologia, cujo produto produz um resíduo energético limpo e flexível no seu uso como também o seu manuseio, é vista como uma das formas de utilização de diferentes tipos de matéria-prima com grande perspectiva para o futuro.
\end{abstract}

\footnotetext{
Abstract The project "Evaluations of operational parameters in a biomass gasifier flow competitor", which began the first week of October 2011, aims to evaluate the influence of different types of biomass and operational parameters of an aerator type flow concurrent (downdraft). Just like, perform sampling and quantitative and qualitative analysis of samples in order to promote improvements in research and thereby enhances this technology. As well as studying the application of the gas produced in internal combustion engines and its use in the electric sector. This research provides a better understanding of the biomass gasification process that is not a recent one, but is continuously growing. This technology, which produces a waste product energy clean and flexible in its use as well as in its management, is seen as one way of using different types of raw material with great prospects for the future
} 


\section{Introdução}

O projeto sobre a gaseificação visa avaliar a influência de três diferentes tipos de matéria-prima (cavaco de madeira, torta de mamona e casca de arroz) e de parâmetros operacionais do gaseificador para a produção do gás utilizado para geração de eletricidade por meio de combustão interna.

Para tal finalidade, foi necessário um levantamento bibliográfico sobre a gaseificação de biomassa por meio de teses de doutorado, livros e seminários vinculados ao tema para a execução do projeto, cujos nomes dos autores se encontram na página de Referências bibliográficas. Após o período de busca, sucedeu o treinamento para a utilização dos equipamentos do laboratório de combustíveis com o propósito de executar os ensaios necessários à

caracterização da matéria-prima. Além de rodadas de gaseificação para a coleta e análise qualitativa e quantitativa do gás gerado.

Essa pesquisa foi realizada no laboratório de combustíveis e energia - POLICOM-UPE, com ajuda de mestrandos, doutores, docentes e discentes que entendem sobre $o$ assunto vigente. Esse projeto é de grande importância para o conhecimento de engenheiros que visam trabalhar na área de combustíveis, pois essa tecnologia que tende a se tornar uma alternativa para a geração de energia elétrica.

\section{Objetivo}

Avaliar a influência dos parâmetros dos diferentes tipos de biomassa e de parâmetros operacionais de um gaseificador do tipo fluxo concorrente (downdraft ventilado) na produção de gás com a finalidade de gerar eletricidade por meio de motor de combustão interna.

Assim como, despertar no aluno iniciante o interesse pela pesquisa, apresentando-o ao método científico e fornecendo base e estímulo necessário à formação de um novo pesquisador.

\section{Metodologia}

\subsection{Estilos}

$1^{\circ}$ trimestre: Levantamento bibliográfico sobre o processo de gaseificação de biomassa através de teses, livros e seminários. E execução de revisão bibliográfica completa sobre $\mathrm{o}$ assunto.

$2^{\circ}$ trimestre: Treinamento na utilização dos equipamentos do laboratório de combustíveis (POLICOM-UPE) para a execução de ensaios necessários à caracterização térmica das biomassas.

$3^{\circ}$ trimestre: Caracterização das biomassas, cavaco de madeira e pellet da torta de mamona, por meio da análise do teor de umidade, análise calorimétrica e termogravimétrica. Execução de rodadas de gaseificação.

$4^{\circ}$ trimestre: Execução de rodadas de gaseificação para avaliação da influência dos parâmetros operacionais do gaseificador. Compilação e análise dos resultados obtidos para a publicação de relatórios e artigos.

\subsection{Material de estudo}

A gaseificação é um processo que transforma combustíveis sólidos ou líquidos (biomassas), podendo ser de origem animal ou vegetal, em combustíveis gasosos (gás de síntese). $\mathrm{O}$ gás produzido contém hidrogênio (H2), monóxido de carbono $(\mathrm{CO})$, metano (CH4), dióxido de carbono entre outros hidrocarbonetos. Essa transformação acontece por meio de reações termoquímicas, podendo utilizar como oxidantes o vapor quente, ar ou oxigênio.

\subsubsection{Gaseificador}

O tipo de gaseificador estudado no projeto de iniciação é o de leito fixo concorrente do tipo "downdraft", instalado na Escola Politécnica de Pernambuco. Esse tipo de reator é um dos mais difundido. O gaseificador "downdraft" é relativamente fácil de ser projetado e manuseado e é eficiente trabalhando com materiais de elevada densidade e granulometria grosseira. Ele é alimentado pelo topo e o ar é alimentado pela lateral do reator, acima da grelha. Seu tipo de acomodação e funcionamento lhe permite uma produção de gás parcialmente livre de alcatrão (hidrocarbonetos poliaromáticos).

O gaseificador de fluxo concorrente apresenta quatro zonas de reações: secagem, pirólise, oxidação e redução, ver Figura 01. A secagem consiste na zona em que ocorre a evaporação da água contida na biomassa utilizando o calor proveniente das zonas inferiores. A velocidade de reação depende da superfície de contato do material entre outros fatores. Nesta fase a temperatura atinge a faixa de 70$200^{\circ} \mathrm{C}$. Na pirolise ocorre a degradação térmica da matéria-prima. Nessa zona as temperaturas estão na faixa de $350-600{ }^{\circ} \mathrm{C}$. Na oxidação, os produtos produzidos na pirolise sofrem oxidação, nessa etapa as reações ocorrem muito rápidas. Essas reações são extremamente exotérmicas, ocasionando o aumento da temperatura que chega a $1100{ }^{\circ} \mathrm{C}$. Na zona de redução, o carvão pirolisado é transformado em gás através de reações com os gases quentes 
oriundos da oxidação. Ver Figura 02, que apresenta os principais processos de conversão térmica e os produtos gerados da pirólise, gaseificação e combustão.
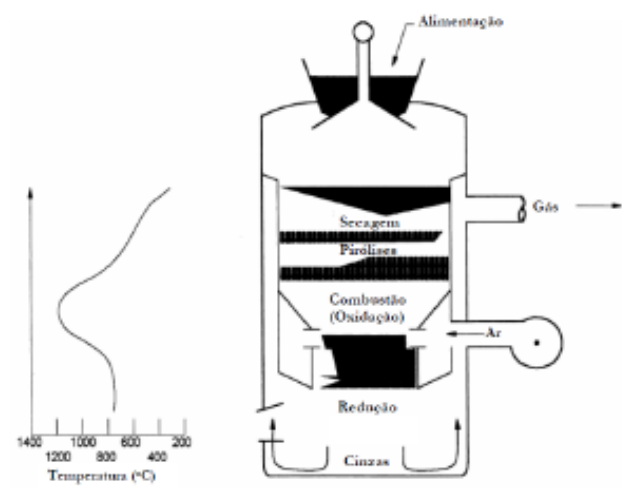

Figura 01: Gaseificador concorrente-downdraft. Fonte: McKendry(2002).

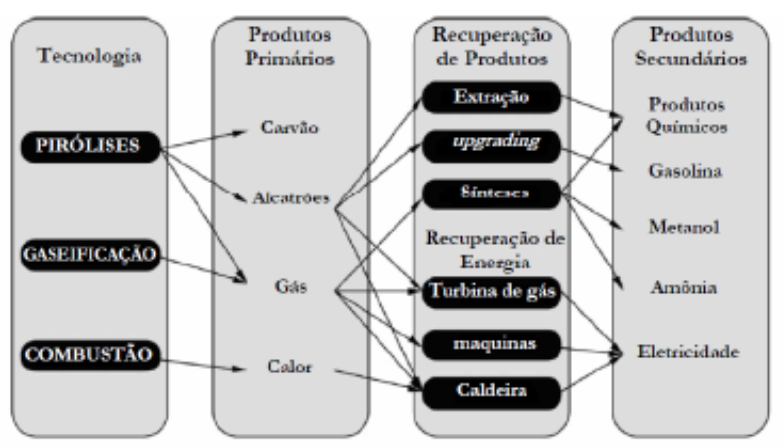

Figura 02: Processos de conversão térmica e os produtos gerados.

Fonte: Desconhecida.

A principal vantagem desse tipo de reator consiste na possibilidade de produzir um gás parcialmente livre de alcatrões, possibilitando ser usado imediatamente em motores. O alcatrão consiste no produto da pirólise. Este produz gases que se dividem em duas categorias: os gases não condensáveis $(\mathrm{CO}, \mathrm{CO} 2$ entre outros) e o alcatrão (hidrocarboneto poli-aromático). A quantidade desse elemento depende da temperatura e da pressão que rege o processo de gaseificação. Ele pode reagir formando materiais voláteis, condensar ou produzir carvão.

\subsubsection{Biomassa}

Uma das biomassas estudadas no projeto de iniciação consiste nos resíduos de madeira, que pode ser encontrada em grande quantidade, tanto nas indústrias quanto nas florestas (devido a sua exploração ilegal). Essa alta quantidade de desperdícios representa um grave problema para o meio ambiente. Sua utilização na geração de energia representa uma excelente alternativa para a recuperação desses rejeitos sólidos.

Já a mamona devido a sua grande produtividade e variedade, principalmente no Brasil, torna-se uma boa fonte de produção energética tanto na área de gaseificação como também na parte de fabricação de biodiesel. Possuindo assim, um elevado aproveitamento da matéria-prima. Para essa pesquisa, foi requerida apenas a torta de mamona que consiste na semente sem o óleo.

Logo, o estabelecimento dessa nova tecnologia representa uma alternativa para recuperação energética dos rejeitos sólidos. Apesar de que, muitos rejeitos não poderem ser utilizados e outros demandam de certo pré-tratamento, porém, há os que podem ser inteiramente aproveitados e submetidos diretamente ao processo de gaseificação, como: rejeitos da indústria do papel, plástico, madeireira e os resíduos da agricultura.

\subsubsection{Impactos}

A utilização da biomassa como fonte de energia, tem gerado grande interesse em virtude da decadência das reservas de combustíveis fósseis, sendo o Brasil o terceiro maior produtor de bio-eletricidade perdendo apenas para os Estados Unidos e a Alemanha segundo pesquisas realizadas pela DEMIRBAS et. al (2005). O estabelecimento dessa nova tecnologia de conversão de matéria-prima é uma grande oportunidade principalmente na geração de eletricidade para regiões isoladas.

Uma das grandes desvantagens da utilização da biomassa consiste na grande demanda de mão- de-obra intensiva para produzir, colher, transportar, pois a mesma se encontra dispersa nas grandes regiões. Porém, essa grande necessidade de mão-de-obra é uma excelente oportunidade para a geração de empregos nas plantações.

Portanto, além dessa tecnologia ser de grande ajuda na área ambiental e socioeconômica, possui seu lado negativo em virtude do alto teor de cinzas. Porém, seus grandes efeitos minimizam as dificuldades, pois a biomassa é considerada o combustível com grande potencialidade no setor elétrico como alternativa em virtude do declínio dos combustíveis fósseis. 


\section{Resultados obtidos}

As biomassas estudadas são constituídas de umidade, fibras (celulose, hemicelulose e lignina) e cinzas. Por isso, sua caracterização se baseia na analise do teor de umidade, da calorimétrica e da termogravimétrica das biomassas. As caracterizações do cavaco de madeira e do pellet da torta de mamona são descritas a seguir, permitindo uma análise quantitativa e qualitativa da matéria-prima empregada na produção de gás para geração de eletricidade por meio de motor de combustão interna (gerador).

\subsection{Umidade}

A umidade da matéria-prima é uma das principais características que prejudicam a composição do gás de síntese. Biomassas que possuem um teor de umidade acima de $15 \%$ torna o gás produzido pobre no que diz respeito a seu poder calorífico. Logo, a eficiência do gás decresce com o aumento do teor de umidade, pois grandes quantidades de energia são gastas na evaporação da água contida na matéria-prima o que reduz a temperatura do gás, ocasionando a redução da velocidade de reação e a eficiência no processo.

Para obter o teor de umidade, uma pequena quantidade de biomassa é triturada e depositada em cima de uma placa de petri (que foi pesada anteriormente obtendo assim a massa do recipiente (Mrecipiente)). Em seguida, faz-se a medição da massa do recipiente em conjunto com a matéria-prima triturada para encontrar a massa da amostra (Mamostra). Após a pesagem a amostra é colocada na estufa por $2 \mathrm{~h}$, na temperatura de $105^{\circ} \mathrm{C}$. Depois, a mesma é novamente pesada para obter-se a massa final (Mfinal).

Após esses procedimentos, realizam-se os cálculos necessários para encontrar o teor de umidade presente na matéria-prima estudada. Como resultado da analise das duas biomassas observadas obtém-se os resultados a seguir.

Tabela 01. Teor de umidade: Cavaco de madeira (Origem: madeireira)

\begin{tabular}{|l|l|l|l|}
\hline Amostra & 01 & 02 & 03 \\
\hline M(recipiente) & $31,7999 \mathrm{~g}$ & $31,1383 \mathrm{~g}$ & $29,8507 \mathrm{~g}$ \\
\hline $\mathrm{M}$ (amostra) & $5,0628 \mathrm{~g}$ & $5,0108 \mathrm{~g}$ & $5,0267 \mathrm{~g}$ \\
\hline M(final) & $36,2483 \mathrm{~g}$ & $35,5452 \mathrm{~g}$ & $34,2613 \mathrm{~g}$ \\
\hline Umidade & $12,1356 \%$ & $12,0519 \%$ & $12,2565 \%$ \\
\hline
\end{tabular}

Tabela 02. Teor de umidade: Pellets da torta de mamona.

\begin{tabular}{|l|l|l|l|}
\hline Amostra & 01 & 02 & 03 \\
\hline M(recipiente) & $31,7993 \mathrm{~g}$ & $31,1389 \mathrm{~g}$ & $29,8507 \mathrm{~g}$ \\
\hline $\mathrm{M}$ (amostra) & $3,3364 \mathrm{~g}$ & $3,5183 \mathrm{~g}$ & $3,2305 \mathrm{~g}$ \\
\hline $\mathrm{M}$ (final) & $34,8892 \mathrm{~g}$ & $34,4171 \mathrm{~g}$ & $32,8498 \mathrm{~g}$ \\
\hline Umidade & $7,3882 \%$ & $6,8224 \%$ & $7,1629 \%$ \\
\hline
\end{tabular}

\subsection{Calorimetria}

O ensaio necessário para medir o poder calorífico da biomassa estudada consiste em submeter à amostra peletizada à bomba calorimétrica, que é um equipamento utilizado para a determinação do poder calorífico de combustíveis sólidos e líquidos. Nesse processo, ocorre a combustão da amostra que libera calor devido à reação. Essa energia (calor) é transferida para água, cuja temperatura anterior já era conhecida. Com essa diferença de temperatura que a água foi submetida, o equipamento realiza os devidos cálculos e fornece o PCS (poder calorífico superior). Pra obter o PCI (poder calorífico inferior) são realizadas as devidas titulações, cujos resultados são digitados no equipamento que realiza os cálculos necessários e fornece o valor do PCI.

Como resultado da analise calorimétrica das duas biomassas estudadas obtém-se os resultados a seguir:

Tabela 03. Calorimetria: Cavaco de madeira (Origem: madeireira)

\begin{tabular}{|l|l|l|l|}
\hline Amostra & Massa & $\begin{array}{l}\text { PCS(poder } \\
\text { calorífico } \\
\text { superior) }\end{array}$ & $\begin{array}{l}\text { PCI(poder } \\
\text { calorífico } \\
\text { inferior) }\end{array}$ \\
\hline 01 & $0,3271 \mathrm{~g}$ & $17458 \mathrm{~J} / \mathrm{g}$ & $17174 \mathrm{~J} / \mathrm{g}$ \\
\hline 02 & $0,3523 \mathrm{~g}$ & $17555 \mathrm{~J} / \mathrm{g}$ & $17293 \mathrm{~J} / \mathrm{g}$ \\
\hline 03 & $0,2893 \mathrm{~g}$ & $17663 \mathrm{~J} / \mathrm{g}$ & $17340 \mathrm{~J} / \mathrm{g}$ \\
\hline
\end{tabular}

Tabela 04: Calorimetria: Pellets da torta da mamona.

\begin{tabular}{|l|l|l|l|}
\hline Amostra & Massa & $\begin{array}{l}\text { PCS(poder } \\
\text { calorífico } \\
\text { superior) }\end{array}$ & $\begin{array}{l}\text { PCI(poder } \\
\text { calorífico } \\
\text { inferior) }\end{array}$ \\
\hline 01 & $0,3930 \mathrm{~g}$ & $19537 \mathrm{~J} / \mathrm{g}$ & $19340 \mathrm{~J} / \mathrm{g}$ \\
\hline 02 & $0,3529 \mathrm{~g}$ & $19443 \mathrm{~J} / \mathrm{g}$ & $19216 \mathrm{~J} / \mathrm{g}$ \\
\hline 03 & $0,3231 \mathrm{~g}$ & $19335 \mathrm{~J} / \mathrm{g}$ & $19073 \mathrm{~J} / \mathrm{g}$ \\
\hline
\end{tabular}

\subsection{Termogravimetrica}

A análise termogravimétrica consiste em determinar a 
variação da massa das amostras em relação à temperatura. Para tal finalidade, foi utilizada uma balança termogravimétrica Shimadzu, modelo DTG- 60. A análise consiste em colocar uma pequena quantidade da amostra em um cadinho. Esse cadinho que já possui a amostra é comparado com outro cadinho similar vazio com o proposito de detectar a diferença de massa existente entre os dois devido ao aumento de temperatura. Nesse equipamento, a biomassa é submetida a temperaturas elevadas (acima de $800^{\circ} \mathrm{c}$ ). O resultado obtido é plotado em gráfico TGA e DTA.

Como resultado da análise termogravimétrica das duas biomassas estudadas obtém-se os seguintes dados:

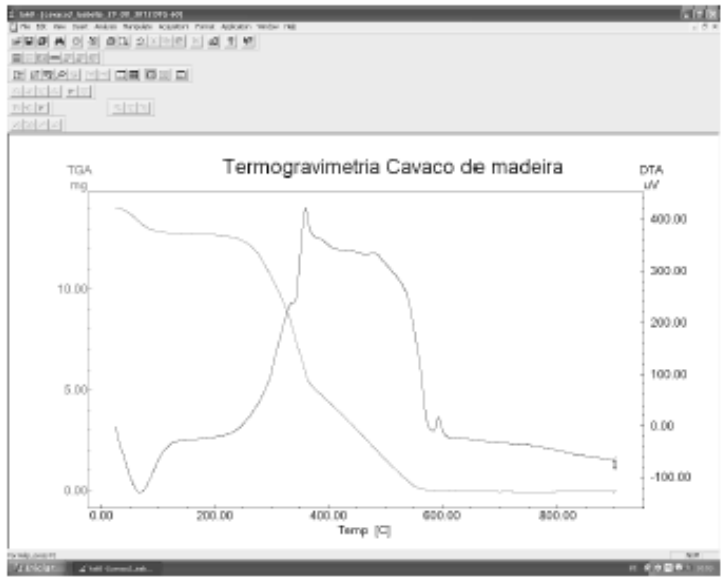

Figura 03: Gráfico 01. Termogravimetria: Cavaco de madeira (Origem: madeireira).

Fonte: Isabella de Andrade Garrett (2012).

Tabela 05. Resultado da análise imediata do cavaco de madeira.

\begin{tabular}{|l|c|}
\hline & $\begin{array}{c}\text { Composição } \\
\text { percentual }\end{array}$ \\
\hline Umidade & 9,24 \\
\hline Material volátil & 90,37 \\
\hline Carbono fixo & - \\
\hline Cinzas $\left(900^{\circ} \mathrm{C}\right)$ & 0,62 \\
\hline
\end{tabular}

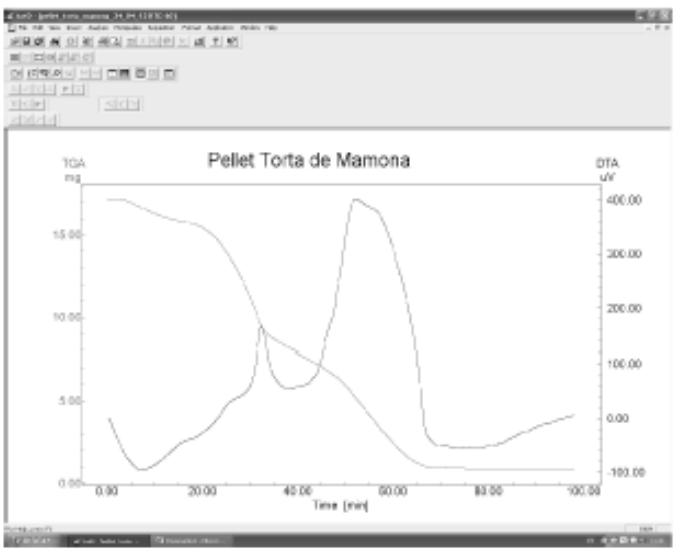

Figura 04: Gráfico 02. Termogravimetria: Pellets da torta da mamona.

Fonte: Isabella de Andrade Garrett (2012).

Tabela6. Resultado da análise imediata do pellet da torta de mamona.

\begin{tabular}{|l|c|}
\hline & $\begin{array}{c}\text { Composição } \\
\text { percentual }\end{array}$ \\
\hline Umidade & 7,85 \\
\hline Material volátil & 86,88 \\
\hline Carbono fixo & 0,14 \\
\hline Cinzas $\left(900^{\circ} \mathrm{C}\right)$ & 4,91 \\
\hline
\end{tabular}

\subsection{Cromatografia}

Devido às adaptações que o gaseificador anda passando, não foi possível analisar os gases produzidos.

\section{Dificuldades encontradas}

O projeto de iniciação "Avaliação de parâmetros operacionais em um gaseificador de biomassa de fluxo concorrente (downdraft ventilado)" passou por algumas dificuldades, principalmente em virtude de que o gaseificador está passando por modificações para agregar o gerador, com a finalidade de adaptar o gás produzido em motores de combustão interna. Como também, está ocorrendo adaptações na sua estrutura para otimizar o seu funcionamento. Por isso, sua operação é mais voltada para testar os equipamentos que o compõem, e assim modifica-lo, do que para analisar o gás obtido. 
Além disso, a localização do gaseificador também fornece um pouco de restrição quanto ao seu uso contínuo, pois o mesmo se encontra ao lado de uma clínica médica. Logo, quando acontecem as rodadas de gaseificação, a queima da biomassa libera muitos poluentes que são arrastados pelo vento e vão ao encontro da clínica. E isso poderá trazer prejuízos a médio prazo ao ambiente vizinho.

Também se pode considerar uma dificuldade devido ao próprio manuseio da máquina, pois requer um grande trabalho braçal para colocá-lo em funcionamento, tanto no transporte da biomassa até o local onde ocorre a queima como também o procedimento para dar o "start-up" para queimar a matéria-prima.

\section{Considerações finais}

O projeto de iniciação "Avaliação de parâmetros operacionais em um gaseificador de biomassa de fluxo concorrente (downdraft ventilado)", visa proporcionar o entendimento de uma tecnologia que tem estado em destaque, pois é vista como uma das formas para a geração de energia elétrica, utilizando diferentes tipos de matériaprima, com maior perspectiva para o futuro.

Essa pesquisa é de grande importância para o desenvolvimento acadêmico do estudante, pois proporciona um interesse pela pesquisa científica através de levantamento bibliográfico sobre a gaseificação de biomassa por meio de teses de doutorado, livros e seminários vinculados ao tema. Além de colocar em contato com pessoas, que entendem muito sobre o assunto e que possuem capacidade técnicas para treinar o estudante na utilização dos equipamentos do laboratório de combustíveis (POLICOM-UPE) com a finalidade de executar os ensaios necessários para à caracterização da matéria-prima.

Logo, o projeto sobre a gaseificação de biomassa foi realizado com sucesso. $\mathrm{O}$ assunto abordado no projeto é de grande valor social, pois a biomassa é considerada o combustível com grande potencialidade para a geração de energia sustentável.

\section{Referências}

[1] Henriques, Rachel Martins. Tese de Doutorado: Potencial para a geração de energia elétrica no Brasil com resíduos de biomassa através da gaseificação. Rio de Janeiro, Rio de Janeiro; UFRJ, 2009.

[2] Andrade, Rubenildo Vieira. Tese de Doutorado: Gaseificação de biomassa: Uma análise teórica e experimental. Itajubá, MG; UFI, 2007.
[3] Barreto, Eduardo José Fagundes. Combustão e gaseificação de biomassa sólida. (soluções energéticas para a Amazonas.). $1^{\circ}$ ed. Ministério de Minas e Energia. Brasília, 2008.

[4] Peres, Sérgio.; Silva, Fabio M. V.; Gusmão, Alexandre D. ; Loureiro, Eduardo C. M.; Azevêdo, Bernardo C.; Júnior, Adalberto F. N.; Almeida, Carlos H. T. Potencial energético da biomassa oriunda de canteiro de obras da construção civil através da gaseificação. Recife,PE,UPE,2012 